\title{
Mecânica Quântica no Espaço de Fase: II. Estados Coerentes
}

\author{
(Quantum Mechanics in Phase Space: II. Coherent States) \\ Marcel Novaes \\ Instituto de Física de São Carlos, Universidade de São Paulo, \\ Caixa Postal 369, 13560-970 São Carlos, SP, Brasil \\ E-mail: marcel@if.sc.usp.br
}

Recebido em 23 de maio, 2002. Aceito em 26 de setembro, 2002.

\begin{abstract}
Apresentamos uma revisão do cálculo dos estados coerentes para um grupo de Lie $G$ compacto como forma de se estabelecer um espaço de fase e uma dinâmica Hamiltoniana (no limite semiclássico) para um sistema quântico com a simetria de $G$. As propriedades desses estados são investigadas no caso geral e nos exemplos tradicionais do oscilador harmônico e do momento angular. O material apresentado foi parte do primeiro mini-curso de verão em física teórica do Instituto de Física de São Carlos, Universidade de São Paulo.
\end{abstract}

We present a review of the coherent state calculation for a compact Lie group $G$ as a way to stablish a phase space and a Hamiltonian dynamics (in the semiclassical limit) for a quantum system with the symmetry of $G$. The properties of these states are investigated in the general case as well as in the traditional examples of the harmonic oscillator and angular momentum. This material was part of the first summer mini-course on theoretical physics at the Instituto de Física de São Carlos, Universidade de São Paulo.

\section{Introdução}

O tema deste curso foi a existência de um espaço de fase "natural" para alguns sistemas quânticos, no qual a equação de Schrödinger seja equivalente às equações de Hamilton no limite $\hbar \rightarrow 0$. O que motiva essa preocupação é a necessidade de se compatibilizar a mecânica clássica com a quântica no sentido de se estabelecer uma transição suave entre elas no limite semiclássico. Uma área importante que se abre nesse processo é a que se dedica a investigar as propriedades de sistemas quânticos cujo análogo clássico é caótico, no que se convencionou chamar "caos quântico" $[1,2]$.

Uma primeira abordagem ao formalismo da quantização foi feita por Bohr e Sommerfeld, que interpretaram a constante de Planck como um quantum de ação. Entretanto, seu formalismo se baseava numa estrutura clássica subjacente que fosse integrável. Einstein foi o primeiro a notar, baseado no trabalho de Poincaré, que a regra de quantização de Bohr e Sommerfeld não deveria ser válida em geral para sistemas com mais de um grau de liberdade, devido à inexistência de toros invariantes em sistemas não-integráveis. A moderna teoria EBK (Einstein-Brillouin-Keller) e o uso de órbitas clássicas na obtenção de propriedades quânticas é muito bem explorado por exemplo em [2]. Outras abordagens modernas ao caos quântico incluem por exemplo a teoria de matrizes aleatórias, as cicatrizes da função de onda e a função de Wigner, que não foram tratados neste curso (o formalismo de Wigner-Weyl foi abordado em [3]). Referências interessantes a esses assuntos são, por exemplo, os artigos de revisão [4] e [5].

Nosso objetivo neste artigo é a "classicalização" usando estados coerentes: baseados no grupo de simetria de um dado sistema quântico, obteremos o sistema clássico correspondente. A seção II aborda os estados coerentes de uma perspectiva histórica e introdutória, tratando dos sistemas paradigmáticos em física teórica: o oscilador harmônico e o momentum angular. A seção III é a mais importante, na qual nos preocupamos com uma teoria geral dos estados coerentes para grupos de Lie compactos. Na seção IV voltamos aos exemplos elementares à luz da teoria geral e apresentamos conclusões na seção V.

As principais referências para o curso foram os artigos de revisão [6] e [7]. Os estados coerentes do campo de radiação são tratados em qualquer livro de ótica quântica (os artigos originais de Roy Glauber [8]). Sobre os estados coerentes do momentum angular, a referência clássica é [9]. Sobre estados coerentes para 
qualquer grupo de Lie, o livro de Perelomov [10] é obrigatório, mas mais avançado. Textos com um pouco mais de detalhes são [11] e [12]; dos mesmos autores, [13] e [14] são mais curtos e tratam casos particulares. Diversos artigos estão reunidos nas coletâneas [15] e [16].

Assumimos um conhecimento prévio do leitor em relação a mecânica quântica básica, incluindo o formalismo de integrais de trajetória de Feynman. Fazemos referência a alguns conceitos da mecânica clássica moderna, como formas diferenciais, que não são geralmente abordados em cursos de graduação, mas mesmo o leitor que não possuir familiaridade com esses conceitos poderá acompanhar a essência da exposição. Álgebras e grupos de Lie são nosso principal objeto de estudo aqui, e acreditamos que os leitores devam estar familiares com os grupos de rotações em duas em três dimensões e suas álgebras.

\section{Casos Particulares}

\section{II.1 Oscilador Harmônico}

\section{II.1.1 Definição e Propriedades}

Os estados coerentes do oscilador harmônico, chamados canônicos, foram descobertos pelo próprio Schrödinger (em 1926), que notou as propriedades semiclássicas desses estados. Isso permaneceu uma curiosidade por muitos anos (principalmente porque não foi possível encontrar estados análogos para o átomo de hidrogênio; essa é uma área de pesquisa, veja por exemplo [17] e as referências aí contidas), até o começo da década de 60 quando Glauber [8] estabeleceu a relação entre esses estados e propriedades do campo de radiação, dando origem ao que hoje se conhece como "ótica quântica". Como nosso interesse é a generalização do conceito de estado coerente, adotaremos aqui uma abordagem algébrica. Os leitores interessados nas implicações em ótica podem consultar por exemplo a Ref. [18].

A álgebra associada ao oscilador harmônico,

$$
\left[a, a^{\dagger}\right]=1, \quad\left[\hat{n}, a^{\dagger}\right]=a^{\dagger},
$$

é gerada pelos operadores $\left\{a, a^{\dagger}, \hat{n}, 1\right\}$ definidos por

$$
a^{\dagger}=\sqrt{\frac{m \omega}{\hbar}} X+i \frac{1}{\sqrt{m \omega \hbar}} P, \quad \hat{n}=a^{\dagger} a,
$$

onde $X$ e $P$ representam a posição e o momentum de uma partícula que se move em uma dimensão.

As relações de comutação acima levam à relação de incerteza $\Delta X \Delta P \geq \frac{\hbar}{2}$, que implica que a partícula não possui valores bem definidos de posição e de momentum simultaneamente $\left(\Delta f=\sqrt{\left\langle(f-\langle f\rangle)^{2}\right\rangle}\right.$ representa o desvio quadrático médio da variável $f$ ). Sendo assim, não é possível representar o sistema como um "ponto" em um espaço de fase, como fazemos tradicionalmente em mecânica clássica.

Essa álgebra é representada por matrizes de dimensão infinita que satisfazem

$$
\hat{n}|n\rangle=n|n\rangle, \quad a|n\rangle=\sqrt{n}|n-1\rangle, \quad a^{\dagger}|n\rangle=\sqrt{n+1}|n+1\rangle, \quad n=0,1,2, \ldots
$$

A Hamiltoniana do oscilador harmônico (medida a partir da energia de ponto zero) é

$$
H=\frac{m}{2}\left(\omega^{2} X^{2}+P^{2}\right)=\hbar \omega \hat{n}
$$

O estado coerente associado a essa álgebra, também chamado estado coerente canônico, é definido como:

$$
|\alpha\rangle=e^{\alpha a^{\dagger}-\alpha^{*} a}|0\rangle=\mathrm{e}^{-|\alpha|^{2} / 2} \mathrm{e}^{\alpha a^{\dagger}}|0\rangle=\mathrm{e}^{-|\alpha|^{2} / 2} \sum_{n=0}^{\infty} \frac{\alpha^{n}}{\sqrt{n !}}|n\rangle, \quad \alpha \in C .
$$

Vemos que a cada ponto do plano complexo corresponde um estado coerente. A seguir as principais propriedades desses estados:

$$
\begin{aligned}
\text { Autoestados do operador de aniquilação } & :|\alpha\rangle=\alpha|\alpha\rangle, \\
\text { Estabilidade } & : e^{-i H t}|\alpha\rangle=\left|\alpha e^{i t}\right\rangle, \\
\text { Mínima incerteza } & : \Delta X \Delta P=\frac{\hbar}{2}, \\
\text { Não-ortogonalidade } & :\langle\alpha \mid \beta\rangle=\exp \left\{-\frac{1}{2}|\alpha|^{2}+\beta \alpha^{*}-\frac{1}{2}|\beta|^{2}\right\} \\
\text { Supercompleteza } & : \frac{1}{\pi} \int d^{2} \alpha|\alpha\rangle\langle\alpha|=1, \\
\text { Variável de ação } & :\langle\alpha|H| \alpha\rangle=\hbar \omega|\alpha|^{2},
\end{aligned}
$$


onde a integral é feita sobre todo o plano complexo. Uma família de estados é dita supercompleta se possuir um subconjunto completo; uma tal família não pode possuir todos seus elementos ortogonais entre si. A não ortogonalidade é uma característica geral dos estados coerentes, assim como a supercompleteza.

Cada uma das propriedades listada acima foi ponto de partida para uma generalização diferente dos estados coerentes. A primeira não pode ser satisfeita em álgebras compactas, mas funciona para $s u(1,1)$, por exemplo [19]. A segunda é uma propriedade bastante desejável: o resultado da evolução de um estado coerente inicial $\left|\alpha_{0}\right\rangle$ é um outro estado coerente $\left|\alpha_{0} e^{i t}\right\rangle$, que no lano complexo corresponde a uma rotação do estado inicial. A relação de mínima incerteza foi usada em uma série de trabalhos, dentre os quais se destacam [20]. A supercompleteza foi a principal propriedade exigida por
Klauder em seu trabalho seminal [21]; recentemente a última foi também reconhecida como interessante [22]. Neste curso usamos a definição de Perelomov [10], que deriva da própria expressão (5).

\section{II.1.2 Integral de trajetória}

Dados dois estados coerentes $\left|\alpha_{0}\right\rangle$ e $\left|\alpha_{N}\right\rangle$, podemos perguntar qual a probabilidade de que uma partícula inicialmente em $\left|\alpha_{0}\right\rangle$ esteja em $\left|\alpha_{N}\right\rangle$ depois de um tempo $t$. Ora, a resposta é dada pelo propagador:

$$
K\left(\alpha_{0}, \alpha_{N}, t\right)=\left\langle\alpha_{N}\left|e^{-\frac{i}{\hbar} H t}\right| \alpha_{0}\right\rangle .
$$

Para calcular essa grandeza, dividimos o intervalo de tempo em $N$ intervalos pequenos de tamanho $\varepsilon=$ $t / N$ e usamos $N-1$ vezes a crucial propriedade $\pi^{-1} \int d^{2} \alpha|\alpha\rangle\langle\alpha|=1$ para obter

$$
K\left(\alpha_{0}, \alpha_{N}, t\right)=\lim _{N \rightarrow \infty} \int \cdots \int \prod_{n=1}^{N-1} \frac{d^{2} \alpha_{n}}{\pi} \prod_{k=1}^{N}\left\langle\alpha_{k}\left|e^{-\frac{i}{\hbar} \varepsilon H}\right| \alpha_{k-1}\right\rangle .
$$

Considerando $\varepsilon \ll 1$, fazemos a aproximação

$$
\left\langle\alpha_{k}\left|e^{-\frac{i}{\hbar} \varepsilon H}\right| \alpha_{k-1}\right\rangle \simeq\left\langle\alpha_{k}\left|1-\frac{i}{\hbar} \varepsilon H\right| \alpha_{k-1}\right\rangle=\left\langle\alpha_{k} \mid \alpha_{k-1}\right\rangle\left(1-\frac{i}{\hbar} \varepsilon \frac{\left\langle\alpha_{k}|H| \alpha_{k-1}\right\rangle}{\left\langle\alpha_{k} \mid \alpha_{k-1}\right\rangle}\right) .
$$

Sabemos que

$$
\left\langle\alpha_{k} \mid \alpha_{k-1}\right\rangle=\exp \left\{-\frac{1}{2}\left|\alpha_{k}\right|^{2}+\alpha_{k-1} \alpha_{k}^{*}-\frac{1}{2}\left|\alpha_{k-1}\right|^{2}\right\}
$$

e nessa expressão somamos e subtraímos os termos $\left|\alpha_{k-1}\right|^{2}, \alpha_{k} \alpha_{k-1}^{*} / 2$ e $\left|\alpha_{k-1}\right|^{2} / 2$. Apóes rearranjar os fatores adequadamente, temos

$$
\left\langle\alpha_{k} \mid \alpha_{k-1}\right\rangle=\exp \left\{-\frac{1}{2} \alpha_{k} \Delta_{k}^{*}-\frac{1}{2} \alpha_{k-1}^{*} \Delta_{k}+\alpha_{k-1} \Delta_{k}^{*}\right\}
$$

onde $\Delta_{k}=\alpha_{k}-\alpha_{k-1}$. Considerando que no limite $\varepsilon \rightarrow 0$ temos

$$
\frac{\left\langle\alpha_{k}|H| \alpha_{k-1}\right\rangle}{\left\langle\alpha_{k} \mid \alpha_{k-1}\right\rangle} \rightarrow \mathcal{H}\left(\alpha_{k}, \alpha_{k}^{*}\right)
$$

fazemos

$$
\left\langle\alpha_{k}\left|e^{-\frac{i}{\hbar} \varepsilon H}\right| \alpha_{k-1}\right\rangle \simeq\left\langle\alpha_{k} \mid \alpha_{k-1}\right\rangle e^{-\frac{i}{\hbar} \varepsilon \mathcal{H}\left(\alpha_{k}, \alpha_{k}^{*}\right)} .
$$

O propagador em (13) fica portanto

$$
\begin{aligned}
K\left(\alpha_{0}, \alpha_{N}, t\right) & =\lim _{\varepsilon \rightarrow 0} \int \cdots \int \prod_{n=1}^{N-1} \frac{d^{2} \alpha_{n}}{\pi} \prod_{k=1}^{N} e^{-\frac{i}{\hbar} S_{k}}=\lim _{\varepsilon \rightarrow 0} \int \cdots \int \prod_{n=1}^{N-1} \frac{d^{2} \alpha_{n}}{\pi} e^{-i S / \hbar}, \\
S & =\sum_{k=1}^{N} i \hbar\left(\frac{1}{2} \alpha_{k} \Delta_{k}^{*}+\frac{1}{2} \alpha_{k-1}^{*} \Delta_{k}-\alpha_{k-1} \Delta_{k}^{*}\right)-\varepsilon \mathcal{H}\left(\alpha_{k}, \alpha_{k}^{*}\right) .
\end{aligned}
$$

Podemos tomar agora o limite para o contínuo, no

qual

$$
S=\int_{0}^{t} i \hbar \frac{\alpha^{*} \dot{\alpha}-\alpha \dot{\alpha}^{*}}{2}-\mathcal{H}\left(\alpha, \alpha^{*}\right) d t
$$


e teremos portanto uma Lagrangeana

$$
\mathcal{L}\left(\alpha, \alpha^{*}\right)=i \hbar \frac{\alpha^{*} \dot{\alpha}-\alpha \dot{\alpha}^{*}}{2}-\mathcal{H}\left(\alpha, \alpha^{*}\right) .
$$

Se notarmos que

$$
\left\langle\alpha\left|\frac{\partial}{\partial \alpha}\right| \alpha\right\rangle=\frac{\alpha^{*}}{2}
$$

$$
\frac{\partial}{\partial t}=\dot{\alpha} \frac{\partial}{\partial \alpha}+\dot{\alpha}^{*} \frac{\partial}{\partial \alpha^{*}}
$$

podemos escrever

$$
\mathcal{L}\left(\alpha, \alpha^{*}\right)=\left\langle\alpha\left|i \hbar \frac{\partial}{\partial t}-H\right| \alpha\right\rangle .
$$

É importante deixar claro que todo o formalismo desenvolvido nesta seção é exclusivamente quântico. As integrações são feitas sobre o espaço dos estados coerentes, não sobre um espaço de fase clássico dado $a$ priori, como no formalismo original de Feynman [23]. Para uma discussão extremamente recente a respeito de integrais de trajetória usando estados coerentes, veja $[24]$.

\section{II.1.3 Limite Semiclássico}

Entendemos como limite semiclássico a situação em que a ação de qualquer trajetória é muito maior que a constante de Planck

$$
S \gg \hbar \text {. }
$$

Nesse caso vale o princípio de mínima ação $\delta S=0$, pois empregamos a aproximação de fase estacionária ao propagador (veja por exemplo [23]). A equação de Lagrange neste caso fornece as equações

$$
\dot{\alpha}=\frac{1}{i \hbar} \frac{\partial \mathcal{H}}{\partial \alpha^{*}}
$$

e sua conjugada. Podemos escrevê-las em termos de um parêntese de Poisson:

$$
\{f, g\}=\frac{1}{i \hbar}\left\{\frac{\partial f}{\partial \alpha} \frac{\partial g}{\partial \alpha^{*}}-\frac{\partial f}{\partial \alpha^{*}} \frac{\partial g}{\partial \alpha}\right\}
$$

e podemos reduzir este parêntese a uma expressão mais familiar definindo $\alpha=(q+i p) / \sqrt{2 \hbar}$. Usando essas novas variáveis teremos

$$
\mathcal{L}(q, p)=\frac{p \dot{q}-q \dot{p}}{2}-\mathcal{H}(q, p)
$$

$\mathrm{e}$

$$
\{f, g\}=\left\{\frac{\partial f}{\partial q} \frac{\partial g}{\partial p}-\frac{\partial f}{\partial p} \frac{\partial g}{\partial q}\right\} .
$$

As equações de movimento (23) tornam-se as equações de Hamilton:

$$
\dot{q}=\frac{\partial \mathcal{H}}{\partial p}, \quad \dot{p}=-\frac{\partial \mathcal{H}}{\partial q},
$$

e portanto o plano complexo é identificado identicamente com o espaço de fase de um sistema unidimensional. O estado coerente pode ser visto como um ponto que se move nesse plano sobre a mesma trajetória do sistema clássico.

Consideramos que as variáveis $\{q, p\}$ descrevem o movimento de uma partícula quântica qualquer. Entretanto, devemos também levar em conta o grau de liberdade intrínseco chamado spin. O spin é algebricamente equivalente a um momento angular, e é disso que trataremos na seção seguinte.

\section{II.2 Momentum Angular}

\section{II.2.1 Definição e Propriedades}

Em mecânica quântica, a cada componente do momentum angular de uma partícula associa-se um operador hermitiano, $J_{x}, J_{y}$ ou $J_{z}$. Com base nesses operadores, que obedecem a relação de comutação $\left[J_{i}, J_{j}\right]=i J_{k}$ $\left(i, j, k\right.$ cíclicos), definimos $J_{ \pm}=J_{x} \pm i J_{y}$ de forma a obtermos as relações de comutação abaixo:

$$
\left[J_{+}, J_{-}\right]=2 J_{z}, \quad\left[J_{z}, J_{ \pm}\right]= \pm J_{ \pm}
$$

Essa álgebra de Lie é denotada $s u(2)$ ou $s o(3)$, e suas representações unitárias irredutíveis são etiquetadas por um número $j$ tal que $2 j \in \mathbb{N}$ e dadas por

$$
\begin{aligned}
J_{z}|j, m\rangle & =m|j, m\rangle \\
J_{ \pm}|j, m\rangle & =\sqrt{(j \mp m)(j \pm m+1)}|j, m \pm 1\rangle
\end{aligned}
$$

Essas relações de comutação levam à relação de incerteza $\Delta J_{x} \Delta J_{y} \geq \frac{\hbar}{2}\left|\left\langle J_{z}\right\rangle\right|$.

O estado coerente, analogamente a (5), é definido por

$$
|z\rangle=\frac{e^{z J_{+}}}{\left(1+|z|^{2}\right)^{j}}|j,-j\rangle=\sum_{m=-j}^{j} \sqrt{\left(\begin{array}{c}
2 j \\
j+m
\end{array}\right)} \frac{z^{j+m}}{\left(1+|z|^{2}\right)^{j}}|j, m\rangle, \quad z \in \mathbb{C}
$$

e possui as seguintes propriedades:

$$
\text { Não-ortogonalidade }:\left\langle z_{1} \mid z_{2}\right\rangle=\frac{\left(1+z_{1}^{*} z_{2}\right)^{2 j}}{\left(1+\left|z_{1}\right|^{2}\right)^{j}\left(1+\left|z_{2}\right|^{2}\right)^{j}},
$$




$$
\begin{array}{rll}
\text { Valores Médios } & : \quad\left\langle z\left|J_{z}\right| z\right\rangle=j \frac{|z|^{2}-1}{|z|^{2}+1}, \quad\left\langle z\left|J_{+}\right| z\right\rangle=\frac{2 j z^{*}}{1+|z|^{2}}, \\
\text { Estabilidade } & : \quad e^{a J_{z}}|z\rangle=e^{-j a}\left|z e^{a}\right\rangle, \\
\text { Mínima incerteza } & : \quad \Delta J_{x} \Delta J_{y}=\frac{\hbar}{2}\left|\left\langle J_{z}\right\rangle\right|, \\
\text { Supercompleteza } & : \quad \int d \mu\left(z, z^{*}\right)|z\rangle\langle z|=1, \quad d \mu\left(z, z^{*}\right)=\frac{2 j+1}{4 \pi} \frac{d z d z^{*}}{\left(1+|z|^{2}\right)^{2}}
\end{array}
$$

Outra maneira de visualizarmos esses estados é através de uma projeção estereográfica do plano sobre a superfície esférica dada por

$$
z=e^{i \varphi} \tan \frac{\theta}{2}
$$

Nesse caso os estados coerentes ficam

$$
|\theta, \varphi\rangle=\sum_{m=-j}^{j} \sqrt{\left(\begin{array}{c}
2 j \\
j+m
\end{array}\right)}(\cos \theta)^{j-m}(\sin \theta)^{j+m} e^{i(j+m) \varphi}|j, m\rangle .
$$

Esta representação tem um apelo geométrico forte, uma vez que o grupo $S O(3)$ (usamos letras minúsculas para designar álgebras, e maiúsculas para designar grupos) está ligado a rotações tridimensionais. Essa relação fica ainda mais evidente quando olhamos o valor médio dos geradores:

$$
\left\langle\theta, \varphi\left|J_{z}\right| \theta, \varphi\right\rangle=j \cos \theta, \quad\left\langle\theta, \varphi\left|J_{x}\right| \theta, \varphi\right\rangle=j \sin \theta \cos \varphi, \quad\left\langle\theta, \varphi\left|J_{y}\right| \theta, \varphi\right\rangle=j \sin \theta \sin \varphi .
$$

Vemos que o estado coerente da álgebra de momento angular se comporta como um vetor clássico de tamanho $j$. Essa superfície esférica é o espaço de fase associado a um sistema de spin $j$.

\section{II.2.2 Integral de trajetória}

O cálculo do propagador neste caso é análogo àquele apresentado para o oscilador harmônico, exceto pelo fato de que agora a medida $d^{2} \alpha / \pi$ deve ser substituída

$$
\begin{aligned}
& \text { por } d \mu\left(z, z^{*}\right) \text { e que } \\
& \qquad\left\langle z_{k} \mid z_{k-1}\right\rangle=\frac{\left(1+z_{k}^{*} z_{k-1}\right)^{2 j}}{\left(1+\left|z_{k}\right|^{2}\right)^{j}\left(1+\left|z_{k-1}\right|^{2}\right)^{j}} .
\end{aligned}
$$

Nessa expressão, somamos e subtraímos $\left|z_{k-1}\right|^{2}$ dentro do numerador de forma que $\left(1+z_{k}^{*} z_{k-1}\right)=(1+$ $\left.\left|z_{k-1}\right|^{2}+\Delta_{k}^{*} z_{k-1}\right)$, onde $\Delta_{k}=z_{k}-z_{k-1}$. No denominador, somamos e subtraímos $\left|z_{k-1}\right|^{2}$ e $z_{k} z_{k-1}^{*}$ de forma que $\left(1+\left|z_{k}\right|^{2}\right)=\left(1+\left|z_{k-1}\right|^{2}+\Delta_{k}^{*} z_{k}+\Delta_{k} z_{k-1}^{*}\right)$. Dessa maneira, temos

$$
\left\langle z_{k} \mid z_{k-1}\right\rangle=\frac{\left(1+\left|z_{k-1}\right|^{2}+\Delta_{k}^{*} z_{k-1}\right)^{2 j}}{\left(1+\left|z_{k-1}\right|^{2}+\Delta_{k}^{*} z_{k}+\Delta_{k} z_{k-1}^{*}\right)^{j}\left(1+\left|z_{k-1}\right|^{2}\right)^{j}} .
$$

Supondo $\Delta_{k}$ da ordem de $\varepsilon$ quando $\varepsilon \rightarrow 0$, expandimos a expressão anterior em série de Taylor

$$
\left\langle z_{k} \mid z_{k-1}\right\rangle \simeq 1+j \frac{\Delta_{k}^{*} z_{k-1}-\Delta_{k} z_{k-1}^{*}}{1+\left|z_{k-1}\right|^{2}} \simeq \exp \left\{j \frac{\Delta_{k}^{*} z_{k-1}-\Delta_{k} z_{k-1}^{*}}{1+\left|z_{k-1}\right|^{2}}\right\}
$$

e é fácil ver que neste caso a Lagrangeana será dada por

$$
\mathcal{L}\left(z, z^{*}\right)=i \hbar j \frac{\left(\dot{z} z^{*}-\dot{z}^{*} z\right)}{1+|z|^{2}}-\mathcal{H}\left(z, z^{*}\right),
$$

e que novamente podemos escrevê-la como $\mathcal{L}\left(z, z^{*}\right)=$ $\left\langle z\left|i \hbar \frac{\partial}{\partial t}-H\right| z\right\rangle$.

Para uma discussão extremamente recente a res- peito de integrais de trajetória usando estados coerentes de $s u(2)$, o leitor pode consultar [25, 26]. Outra boa referência, que inclui uma discussão sobre $s u(1,1)$, 
é [27].

\section{II.2.3 Limite Semiclássico}

Nesse caso a equação de Lagrange fornece

$$
\dot{z}=-i \frac{\left(1+|z|^{2}\right)^{2}}{2 j \hbar} \frac{\partial \mathcal{H}}{\partial z^{*}}
$$

e sua conjugada. Podemos escrevê-las novamente em termos de um parêntese de Poisson:

$$
\{f, g\}=\frac{1}{i \hbar} \frac{\left(1+|z|^{2}\right)^{2}}{2 j}\left\{\frac{\partial f}{\partial z} \frac{\partial g}{\partial z^{*}}-\frac{\partial f}{\partial z^{*}} \frac{\partial g}{\partial z}\right\},
$$

ou, usando as variáveis da projeção estereográfica,

$$
\{f, g\}=\frac{1}{\hbar j \sin \theta}\left\{\frac{\partial f}{\partial \varphi} \frac{\partial g}{\partial \theta}-\frac{\partial f}{\partial \theta} \frac{\partial g}{\partial \varphi}\right\} .
$$

Novamente podemos reduzir essa expressão a uma forma mais familiar, definindo

$$
\frac{q+i p}{\sqrt{4 \hbar j}}=\frac{z}{\sqrt{1+|z|^{2}}}
$$

ou inversamente

$$
z=\frac{q+i p}{\sqrt{4 \hbar j-q^{2}-p^{2}}} .
$$

Note que a relação

$$
q^{2}+p^{2}=4 \hbar j \frac{|z|^{2}}{1+|z|^{2}}
$$

obtida a partir de (47) impõe a desigualdade

$$
0 \leq q^{2}+p^{2} \leq 4 \hbar j .
$$

Usando essas novas variáveis temos novamente

$$
\mathcal{L}(q, p)=\frac{p \dot{q}-q \dot{p}}{2}-\mathcal{H}(q, p)
$$

e

$$
\{f, g\}=\left\{\frac{\partial f}{\partial q} \frac{\partial g}{\partial p}-\frac{\partial f}{\partial p} \frac{\partial g}{\partial q}\right\} .
$$

É importante frisar que neste caso o espaço de fase não é simplesmente $\mathbb{R}^{\not ⿰}$ devido à restrição (50). O espaço de fase mais natural para este sistema é a superfície esférica. Podemos utilizar o plano complexo se o entendermos como advindo de uma projeção estereográfica, mas então a métrica neste plano será nãotrivial como vemos em (45). Outro aspecto essencial é o fato de que nos resultados finais a constante de Planck $\hbar$ aparece sempre multiplicada por $j$. Isso indica que o limite $\hbar \rightarrow 0$ deve ser acompanhado por $j \rightarrow \infty$ de forma que $\hbar j$ seja mantido constante. Veremos mais adiante que esse é realmente o caso.

Tendo visto em detalhe a construção dos estados coerentes e do espaço de fase quântico para os casos mais simples, passamos agora a uma análise mais geral.

\section{Caso Geral}

Consideraremos como caso geral o cálculo dos estados coerentes para uma representação qualquer de um dado grupo de Lie compacto (o oscilador harmônico não se encaixa nesta restrição mas pode ser considerado um caso limite [9]). O espaço de fase natural para um sistema quântico, que no caso do oscilador harmônico é o plano complexo e no caso do momentum angular é a superfície esférica, é dado pelo quociente de seu grupo de simetria $G$ por um subgrupo $H$. Veremos a seguir a definição de espaço quociente e também, de forma sucinta, a decomposição de Cartan de uma álgebra de Lie. Veremos também que $H$ deve ser o subgrupo de isotropia do estado fundamental do sistema.

\section{III.1 Espaço quociente}

Seja $H$ um subgrupo do grupo $G$. Entendemos $g H$ como o conjunto de elementos obtido multiplicando-se todos os elementos de $H$ por $g$, ou seja, $g H=\{g h \mid h \in$ $H\}$. É fácil ver que dados dois elementos $g_{i}, g_{j}$ de $G$ os conjuntos $g_{i} H$ e $g_{j} H$ coincidem ou são disjuntos. Esses conjuntos são chamados cosets (à esquerda) de $G$ por $H$, e sua união é representada por $G / H=\{g H \mid g \in G\}$, chamado espaço coset ou espaço quociente de $G$ por $H$. Note que cada conjunto $g H$ constitui um único elemento do espaço quociente.

Exemplo: Se $G=\mathbb{R}^{*}$ é o grupo dos números reais sem o zero com operação de multiplicação, e se $H=\{-1,1\}$ é o subgrupo de dois elementos chamado $Z_{2}$, então $G / H$ é $\mathbb{R}^{*} / \mathbb{Z}_{\not \nvdash}=\mathbb{R}^{+}$, o conjunto dos números reais positivos (que também constituem um grupo, apesar de esse não ser o caso geral dos espaços quociente).

\section{III.2 Decomposição de Cartan}

Seja $A$ uma álgebra de Lie tal que $e^{A}=G$. A decomposição de Cartan de $A$ é [29]

$$
\left[H_{i}, H_{j}\right]=0, \quad\left[H_{i}, E_{\alpha}\right]=\alpha_{i} E_{\alpha}, \quad\left[E_{\alpha}, E_{-\alpha}\right]=\alpha^{i} H_{i}, \quad\left[E_{\alpha}, E_{\beta}\right]=N_{\alpha \beta} E_{\alpha+\beta} .
$$


Os operadores $H_{i}$ compõem a chamada subálgebra (comutativa) de Cartan. $E_{\alpha}$ e $E_{-\alpha}$ são chamados operadores de levantamento e abaixamento, respectivamente. As outras grandezas são números complexos. No caso do momentum angular, $J_{z}$ é o único componente da subálgebra de Cartan, enquanto que $J_{+}$e $J_{-}$são os operadores de levantamento e abaixamento.

Vamos denotar por $\left|\psi_{0}\right\rangle$ o vetor "mais baixo" (também chamado peso mínimo ou estado fundamental) de uma dada representação unitária de $A$ :

$$
E_{-\alpha}\left|\psi_{0}\right\rangle=0, \quad \text { para todo } \alpha \text {. }
$$

Além de ser aniquilado por todos os operadores de abaixamento, o estado fundamental pode eventualmente sêlo também por alguns dos de levantamento:

$$
E_{\beta}\left|\psi_{0}\right\rangle=0, \quad \text { para alguns } \beta .
$$

Com relação à subálgebra de Cartan, temos

$$
H_{i}\left|\psi_{0}\right\rangle=\left\{0, \Xi\left|\psi_{0}\right\rangle,\right.
$$

onde $\Xi$ é um número real, que depende apenas da dimensão da representação em questão.
A união de todos os operadores $E_{\beta}$ que satisfazem (55) com seus conjugados $E_{-\beta}$ e com a subálgebra de Cartan gera uma álgebra, h. A exponencial dessa álgebra, $H_{0}=e^{\mathfrak{h}}$, é chamado o subgrupo de isotropia do vetor $\left|\psi_{0}\right\rangle$, uma vez que $h\left|\psi_{0}\right\rangle=e^{i \gamma}\left|\psi_{0}\right\rangle$ para todo $h \in H_{0}$.

\section{III.3 Os estados coerentes}

A entidade que nos interessa aqui é o quociente, $C$, do grupo de simetria do sistema pelo subgrupo de isotropia do estado fundamental. Levando em conta a decomposição de Cartan pode-se mostrar que o espaço quociente é obtido através de uma aplicação exponencial:

$$
C(\tau)=G / H_{0}=\exp \left\{\sum_{\alpha} \tau_{\alpha} E_{\alpha}-\tau_{\alpha}^{*} E_{-\alpha}\right\},
$$

onde a soma não inclui os índices $\beta$ contidos em (55). Os estados coerentes serão dados pela ação de elementos desse espaço no estado fundamental:

$$
\left.|\mathbf{z}\rangle=C(\boldsymbol{\tau})\left|\psi_{0}\right\rangle=\frac{1}{\sqrt{N\left(\mathbf{z}, \mathbf{z}^{*}\right)}} e^{\sum_{\gamma} z_{\gamma} E_{\gamma}}\left|\psi_{0}\right\rangle=\frac{1}{\sqrt{N\left(\mathbf{z}, \mathbf{z}^{*}\right)}} \| \mathbf{z}\right\rangle .
$$

Note que a unitariedade do operador $C(\boldsymbol{\tau})$ garante a normalização dos estados $|\mathbf{z}\rangle$.

A relação

$$
\exp \left\{\sum_{\alpha} \tau_{\alpha} E_{\alpha}-\tau_{\alpha}^{*} E_{-\alpha}\right\}\left|\psi_{0}\right\rangle=\frac{1}{\sqrt{N\left(\mathbf{z}, \mathbf{z}^{*}\right)}} \exp \sum_{\gamma} z_{\gamma} E_{\gamma}\left|\psi_{0}\right\rangle
$$

é garantida pela chamada decomposição de Gauss, que garante que toda matriz pode ser escrita como o produto de uma matriz triangular superior, uma diagonal e uma triangular inferior (a relação entre $\boldsymbol{\tau}$ e $\mathbf{z}$ está dada em $(70))$.

\section{III.4 Propriedades}

\section{III.4.1 Supercompleteza}

Os estados coerentes são supercompletos:

$$
\int_{G / H} d \mu_{H}\left(z, z^{*}\right)|z\rangle\left\langle z \mid=\int_{G / H} d \mu\left(z, z^{*}\right) \| z\right\rangle\langle z \|=1
$$

(deixamos o negrito de lado) onde $d \mu_{H}\left(z, z^{*}\right)$ é a medida de Haar sobre $G / H$ e vale a relação

$$
d \mu_{H}\left(z, z^{*}\right)=N\left(z, z^{*}\right) d \mu\left(z, z^{*}\right)
$$

A supercompleteza implica que qualquer estado quântico pode ser escrito como combinação linear de estados coerentes:

$$
\left.|\psi\rangle=\int_{G / H} d \mu\left(z, z^{*}\right) f\left(z^{*}\right) \| z\right\rangle, \quad f\left(z^{*}\right)=\langle z \| \mid \psi\rangle .
$$

As funções $f(z)$ formam um espaço de Hilbert quadrado integrável $L^{2}(G / H)$. A unicidade da expansão (62) implica em

$$
f(x)=\int_{G / H} K\left(x, y^{*}\right) f(y) d \mu(y)
$$

onde a função $K\left(x, y^{*}\right)=\langle y\|\| x\rangle$ é um núcleo reprodutor, análogo à função delta de Dirac. É óbvio que $N\left(z, z^{*}\right)=K\left(z, z^{*}\right)$. Esta importante função é, para uma álgebra de Lie semisimples que satisfaz a decomposição de Cartan, sempre dada por

$$
K\left(z, z^{*}\right)=\operatorname{det}\left(I \pm Z Z^{\dagger}\right)^{ \pm \Xi}
$$

(onde $Z$ é o vetor das variáveis $\mathbf{z}$ ), sendo $\Xi$ definido por (56). 
A supercompleteza permite que escrevamos, para um dado operador $A$,

$$
A=\int_{G / H} \mathcal{A}\left(z, z^{*} ; \tilde{z}, \tilde{z}^{*}\right)|z\rangle\langle\tilde{z}| d \mu_{H}(z) d \mu_{H}(\tilde{z}),
$$

e essa expressão pode ser colocada em uma forma diagonal, chamada representação-P do operador:

$$
A\left(z, z^{*}\right)=\int A_{P}|z\rangle\langle z| d \mu_{H}(z) .
$$

Entretanto, esta representação em geral não é única.

\section{III.4.2 Métrica}

A métrica no espaço coset pode ser escrita explicitamente em termos da grandeza $K\left(z, z^{*}\right)$ [30]

$$
g_{\mu \nu}=\frac{\partial^{2}}{\partial z_{\mu} \partial z_{\nu}^{*}} \ln \left[K\left(z, z^{*}\right)\right] .
$$

Variedades nas quais a métrica deriva de uma função (potencial) são ditas Kahlerianas, e a função $\ln \left[K\left(z, z^{*}\right)\right]$ é o potencial de Kahler neste caso.

A métrica induz de forma natural uma 2-forma fechada não-degenerada [31]

$$
\omega^{2}=i \hbar \sum_{\mu \nu} g_{\mu \nu} d z^{\mu} \wedge d z^{\nu}
$$

e um parêntese de Poisson sobre $G / H$ dado por

$$
\{u, v\}=\frac{1}{i \hbar} \sum_{\mu \nu} g^{\mu \nu}\left\{\frac{\partial u}{\partial z_{\mu}} \frac{\partial v}{\partial z_{\nu}^{*}}-\frac{\partial u}{\partial z_{\nu}^{*}} \frac{\partial v}{\partial z_{\mu}}\right\}
$$

onde $u$ e $v$ são funções definidas sobre $G / H$ e $g^{\mu \nu} g_{\nu \gamma}=$ $\delta_{\gamma}^{\mu}$.

Essa expressão pode sempre ser trazida a uma forma mais tradicional introduzindo-se as funções

$$
\frac{q_{i}+i p_{i}}{\sqrt{2 \hbar \Xi}}=\frac{z_{i}}{\sqrt{1+|\mathbf{z}|^{2}}}=\frac{\tau_{i} \sin |\boldsymbol{\tau}|}{|\boldsymbol{\tau}|}
$$

(onde $|\mathbf{z}|^{2}=\sum\left|z_{i}\right|^{2}$ ) o que leva a

$$
\begin{aligned}
\omega^{2} & =\sum_{i} d q^{i} \wedge d p^{i} \\
\{f, g\} & =\sum_{i, j} \frac{\partial f}{\partial q_{i}} \frac{\partial g}{\partial p_{i}}-\frac{\partial f}{\partial p_{i}} \frac{\partial g}{\partial q_{i}} .
\end{aligned}
$$

\section{III.5 Discussão}

Encontramos portanto uma maneira natural de se introduzir um espaço de fase na mecânica quântica. Dado um sistema com grupo de simetria $G$ e dada uma função dos geradores da álgebra associada $\hat{F}\left[H_{i}, E_{\alpha}\right]$, podemos escrever uma função de coordenadas canônicas $f(\mathbf{q}, \mathbf{p})$ (muitas vezes chamada representação-Q do operador $\hat{F}$ ) sobre o espaço de fase $G / H$ fazendo

$$
f(\mathbf{q}, \mathbf{p})=\langle\mathbf{z}|\hat{F}| \mathbf{z}\rangle .
$$

Resta saber qual a relação entre a dinâmica dessas funções e a dinâmica quântica. Pode-se mostrar que, sendo $A$ e $B$ dois elementos da álgebra, o comutador e o parêntese de Poisson estão relacionados por:

$$
\langle\mathbf{z}|[A, B]| \mathbf{z}\rangle=i \hbar\{\mathcal{A}, \mathcal{B}\}
$$

onde $\mathcal{A}=\langle\mathbf{z}|A| \mathbf{z}\rangle$. Portanto, se a Hamiltoniana for linear, teremos, na representação de Heisenberg:

$$
\frac{d}{d t} \mathcal{A}\left(z, z^{*}\right)=\left\{\mathcal{A}\left(z, z^{*}\right), \mathcal{H}\left(z, z^{*}\right)\right\}
$$

que é simplesmente a equação de Liouville sobre $G / H$. Vemos então que neste caso a dinâmica no espaço de fase quântico é equivalente à dinâmica quântica exata.

Para analisarmos o caso não-linear, definimos observáveis físicos como funções dos geradores da álgebra. Esses geradores são adimensionais e portanto acrescentamos a constante de Planck:

$$
A(\hbar \vec{T})=\sum_{i j} f_{i j}\left(\hbar T_{i}\right)\left(\hbar T_{j}\right)
$$

onde $f_{i j}$ são coeficientes e $T_{i}$ representa qualquer gerador, cuja representação-Q é denotada por $\mathcal{T}_{i}=\left\langle\mathbf{z}\left|T_{i}\right| \mathbf{z}\right\rangle$.

Pode-se mostrar que

$$
\mathcal{A}=A(\hbar \overrightarrow{\mathcal{T}})+\delta A=\sum_{i j} f_{i j} \hbar \mathcal{T}_{i}(\mathbf{q}, \mathbf{p}, \hbar \Xi) \hbar \mathcal{T}_{j}(\mathbf{q}, \mathbf{p}, \hbar \Xi)+\frac{1}{\Xi} f(\mathbf{q}, \mathbf{p}, \hbar \Xi)
$$

ou seja, a representação- $Q$ de um produto de geradores é dada pelo produto das representações- $Q$ individuais mais um termo de correção, $\delta A=\Xi^{-1} f(\mathbf{q}, \mathbf{p}, \hbar \Xi)$, chamado de correlação quântica.
Vemos que no limite $\Xi \rightarrow \infty, \hbar \rightarrow 0$, tal que $\hbar \Xi$ é mantido fixo, a correlação quântica vai a zero e os observáveis quânticos se reduzem às suas contrapartes clássicas. Como já havíamos mencionado, a solução 
para o problema de classicalização, ou seja o limite $\hbar \rightarrow 0$, deve ser encarado como concomitante à divergência do índice $\Xi$ correspondente.

Voltamos agora brevemente aos casos particulares já apresentados, a fim de ver como estão relacionados à teroia geral.

\section{Exemplos Revisitados}

\section{IV.1 Oscilador Harmônico}

O subgrupo de isotropia do estado fundamental, $\left|\psi_{0}\right\rangle \equiv|0\rangle$, é gerado pela identidade e pelo operador $\hat{n}$. Sendo assim, o espaço coset é, neste caso, dado por

$$
D(\alpha)=\mathrm{e}^{\alpha a^{\dagger}-\alpha^{*} a} .
$$

Uma vez que $\left[a,\left[a, a^{\dagger}\right]\right]=\left[a^{\dagger},\left[a, a^{\dagger}\right]\right]=0$, podemos utilizar a fórmula de Baker-Campbell-Hausdorff

$$
e^{A+B}=e^{A} e^{B} e^{-[A, B] / 2},
$$

obtendo

$$
D(\alpha)=\mathrm{e}^{-|\alpha|^{2} / 2} \mathrm{e}^{\alpha a^{\dagger}} \mathrm{e}^{-\alpha^{*} a} .
$$

Os estados coerentes são, portanto, obtidos através da aplicação do operador $D(\alpha)$ ao vácuo. Tal operador é chamado de deslocamento, pois

$$
D(\alpha) a D^{-1}(\alpha)=a+\alpha .
$$

O potencial de Kahler é obtido de forma trivial. A relação

$$
|\alpha\rangle=\mathrm{e}^{-|\alpha|^{2} / 2} \mathrm{e}^{\alpha a^{\dagger}}|0\rangle
$$

leva a

$$
g=\frac{\partial^{2}}{\partial \alpha \partial \alpha^{*}} \ln \left[\mathrm{e}^{|\alpha|^{2}}\right]=1 .
$$

Note que o potencial de Kahler neste caso não possui a forma geral (64). Isso acontece porque a álgebra do oscilador harmônico não é simples.

O parêntese de Poisson será

$$
\{u, v\}=\frac{1}{i \hbar} \sum_{\mu \nu}\left\{\frac{\partial u}{\partial \alpha} \frac{\partial v}{\partial \alpha^{*}}-\frac{\partial u}{\partial \alpha^{*}} \frac{\partial v}{\partial \alpha}\right\},
$$

como já visto.

\section{IV.2 Momentum Angular}

Neste caso, o subgrupo de isotropia é gerado por uma das componentes do momentum angular (a subálgebra de Cartan), por exemplo $J_{z}$. O estado fundamental é o autovetor de $J_{z}$ que possui menor autovalor, $\left|\psi_{0}\right\rangle \equiv|j,-j\rangle$. O grupo $e^{\theta J_{z}}$, que corresponde a rotações em um plano, é denotado $U(1)$ ou $S O(2)$. Logo, o espaço coset adequado neste caso é

$$
S U(2) / U(1) \sim S O(3) / S O(2) \sim S^{2},
$$

que corresponde a uma superfície esférica [7].

A definição dos estados coerentes de $s u(2)$ é

$$
|j, \tau\rangle=e^{\tau J_{+}-\tau^{*} J_{-}}|j,-j\rangle .
$$

A decomposição de Gauss nesse caso é

$$
\exp \left\{\tau J_{+}-\tau^{*} J_{-}\right\}=\exp \left\{z J_{+}\right\} \exp \left\{w J_{z}\right\} \exp \left\{-z^{*} J_{-}\right\}
$$

com

$$
\frac{z}{\sqrt{1+|z|^{2}}}=\frac{\tau \sin |\tau|}{|\tau|}, \quad w=-2 \ln \cos |\tau|,
$$

o que nos leva à definição equivalente (31). Podemos obter a métrica sobre a esfera a partir do potencial de Kahler:

$$
g=\frac{\partial^{2}}{\partial z \partial z^{*}} \ln \left[\left(1+|z|^{2}\right)^{2 j}\right]=\frac{2 j}{\left(1+|z|^{2}\right)^{2}},
$$

e aíestá embutido o parêntese de Poisson

$$
\{f, g\}=\frac{1}{\hbar j \sin \theta}\left\{\frac{\partial f}{\partial \varphi} \frac{\partial g}{\partial \theta}-\frac{\partial f}{\partial \theta} \frac{\partial g}{\partial \varphi}\right\}
$$

Vemos que neste caso $\Xi=2 j$ e assim reobtemos a transformação de coordenadas dada por (47). O limite clássico corresponde a $j \rightarrow \infty, \hbar \rightarrow 0, \operatorname{com} j \hbar=J$ mantido fixo (a existênca de um limite clássico para sistemas de spins foi demonstrada elegantemente em [34]). A correlações quânticas para funções quadráticas dos $J_{i}$ são

$$
\begin{aligned}
\delta\left(\hbar J_{z}\right)^{2} & =\frac{1}{4 \Xi}\left(q^{2}+p^{2}\right)\left(4 j \hbar-q^{2}-p^{2}\right), \\
\delta\left(\hbar J_{+}\right)^{2} & =-\frac{1}{4 \Xi}(q-i p)^{2}\left(4 j \hbar-q^{2}-p^{2}\right), \\
\delta\left(\hbar J_{z} \hbar J_{+}\right) & =\frac{1}{4 \Xi}(q-i p)\left(4 j \hbar-q^{2}-p^{2}\right) \sqrt{4 j \hbar-q^{2}-p^{2}}, \\
\delta\left(\hbar J_{-} \hbar J_{+}\right) & =\frac{1}{4 \Xi}\left(4 j \hbar-q^{2}-p^{2}\right)^{2} .
\end{aligned}
$$


A análise de correlações quânticas em sistemas de spin é feita por exemplo em [35].

\section{Conclusão}

Recentes desenvolvimentos nas áreas de controle quântico, física mesoscópica, computação quântica, eletrodinâmica em cavidades, etc., têm aumentado a preocupação da comunidade com áreas que há algumas décadas eram consideradas de interesse apenas acadêmico, como quantização de sistemas classicamente caóticos, limite semiclássico e afins. Os estados coerentes, assim como as quase-distribuições de probabilidade (e.g. funções de Wigner e Husimi), têm se mostrado ferramentas importantes nessas áreas (como em outras mais tradicionais), e a compreensão de sua conexão com a teoria clássica das simetrias de Lie foi fundamental no desenvolvimento dessas estruturas. Os grupos e as álgebras de Lie se mostraram relevantes em inúmeras áreas da física teórica, e certamente fornecerão elementos importantes às futuras investigações. Este artigo se propõe a uma abordagem introdutória dessas questões, buscando motivar o interesse dos leitores não-especialistas.

\section{Agradecimentos}

Agradeço ao Prof. Esmerindo Bernardes e ao colega Marcelo Marchiolli pela organização e pela participação no primeiro mini-curso de verão em física teórica do IFSC-USP. Agradeço ainda ao Prof. José Eduardo Hornos pela orientação e a um parecerista anônimo pelas valiosas recomendações referentes à apresentação deste trabalho.

\section{Referências}

[1] F. Haake, Quantum signatures of chaos, Springer (2001).

[2] M. C. Gutzwiller, Chaos in quantum and classical mechanics, Springer (1990).

[3] M. A. Marchiolli, Mecânica quântica no espaço de fase: I. Formulação de Weyl-Wigner. Rev. Bras. Ens. Fís. 24(4), 421 (2002)

[4] B. Eckhardt, Quantum mechanics of classicaly nonintegrable systems. Phys. Rep. 163 (4), 205 (1988).

[5] H-W. Lee, Theory and application of the quantum phase-space distribution functions. Phys. Rep. 259 (3), 147 (1995).

[6] W. M. Zhang, D. H. Feng, Quantum nonintegrability in finite systems. Phys. Rep. 252, 1 (1995).

[7] W. M. Zhang, D. H. Feng, R. Gilmore, Coherent states: Theory and some applications. Rev. Mod. Phys. $62,867(1990)$
[8] R. Glauber, The quantum theory of optical coherence. Phys. Rev. 130 (6), 2529 (1963). Coherent and incoherent states of the radiation field. Phys. Rev. 131 (6), 2766 (1963).

[9] F. T. Arecchi, E. Courtens, R. Gilmore, H. Thomas, Atomic coherent states in quantum optics, Phys. Rev. A 6, 2211 (1972).

[10] A. Perelomov, Generalized Coherent States and Their Applications, Springer (1986).

[11] W. M. Zhang, D. H. Feng, J. M. Yuan, S. W. Wang, Integrability and nonintegrability of quantum systems: Quantum integrability and dynamical symmetry. Phys. Rev. A 40 (1), 438 (1989).

[12] W. M. Zhang, D. H. Feng, J. M. Yuan, Integrability and nonintegrability of quantum systems II. Dynamics in quantum phase space. Phys. Rev. A 42 (12), 7125 (1990).

[13] W. M. Zhang, C. C. Martens, D. H. Feng, J. M. Yuan. Dynamical Symmetry Breaking and Quantum Nonintegrability. Phys. Rev. Lett. 61, 2167 (1988).

[14] W. M. Zhang, D. H. Feng, Geometry in quantum nonintegrability. Mod. Phys. Lett A, Vol 8, No 15, 1417 (1993).

[15] J. R. Klauder, B-S. Skagerstam (Ed.), Coherent states: Applications in physics and mathematical physics, World Scientific (1985).

[16] D.H. Feng, J.R. Klauder, M.R. Strayer (Ed.), Coherent states: Past, present and future, World Scientific (1994).

[17] M. G. A. Crawford, Temporally stable coherent states in energy-degerate systems: The hydrogen atom. Phys. Rev. A 62, 012104 (2000).

[18] M. O. Scully, M. S. Zubairy, Quantum optics, Cambridge University Press (1997).

[19] A. O. Barut, Girardello L, New coherent states associated with non-compact groups. Comm. Math. Phys 21, 41 (1971).

[20] M. M. Nieto et all, Coherent states for general potentials I-VI. O número VI está em Phys. Rev. D 23, 927933 (1981).

[21] J. R. Klauder, Continuous-Representation Theory. I. Postulates of continuous-representation theory; II. Generalized relation between quantum and classical dynamics. J. Math. Phys. 4, 1058 (1963).

[22] J-P. Gazeau, J. R. Klauder, Coherent states for systems with discrete and continuous spectrum. J. Phys. A: Math. Gen. 32, 123 (1999).

[23] R. P. Feynman, A. R. Hibbs, Quantum mechanics and path integrals, McGraw-Hill (1965).

[24] M. Baranger, M. A. M. Aguiar, F. Keck, H. J. Korsch, B. Schellhaa $\beta$, Semiclassical approximations in phase space with coherent states. J. Phys. A: Math. Gen. 34, 7227 (2001).

[25] E. A. Kochetov, SU(2) coherent state path integral. J. Math. Phys. 36 (9), 4667 (1995).

[26] M. Stone, K-S. Park, A. Garg, The semiclassical propagator for spin coherent states. J. Math. Phys. 41 (12), 8025 (2000). 
[27] A. Inomata, H. Kuratsuji, C. C. Gerry, Path integrals and coherent states of $S U(2)$ and $S U(1,1)$, World Scientific, 1992.

[28] R. Gilmore, Lie groups, Lie algebras, and some of their applications, Krieger editora (1994).

[29] B. G. Wybourne, Classical groups for physicists , Wiley-Interscience, New York (1974).

[30] S. Helgason, Differential Geometry, Lie Groups and Symmetric Spaces, Academic Press, New York (1978).

[31] V. I. Arnold, Mathematical methods of classical mechanics, Mir Moscow (1987).
[32] J. R. Klauder, Path integral and stationary phase aproximations. Phys. Rev. D 19 (8), 2349 (1979).

[33] E. A. Kochetov, Path integral over the generalized coherent states. J. Math. Phys. 36 (4), 1666 (1995); Quasiclassical path integral in coherent state manifolds. J. Phys. A: Math. Gen, 31, 4473 (1998).

[34] E. H. Lieb, The classical limit of quantum spin systems. Commun. Math. Phys. 31, 615 (1973).

[35] W. M. Zhang, J. M. Yuan, D. H. Feng, Q. Pan, J. Tjon, Quantum fluctuations in classical chaos. Phys. Rev A 42 (6), 3646 (1990). 